\title{
Massive systemic-to-pulmonary collateral arteries in the setting of a cavopulmonary shunt and pulmonary venous stenosis
}

Lars Grosse-Wortmann, Robert Hamilton, Shi-Joon Yoo

doi: $10.1017 /$ S1047951107000960

Cardiology in the Young, Volume 17, Issue 05, Oct 2007, pp 548-550

First published online by Cambridge University Press 19 July 2007

The first author of the above article failed to acknowledge his cross-appointment with the RWTH University of Aachen Germany. At the time of manuscript submission, Lars Grosse-Wortmann was employed by the University of Aachen which funded his research fellowship at the Hospital for Sick Children, Toronto, Canada. The correct affiliation should therefore have read:

Lars Grosse-Wortmann, ${ }^{1,2,3}$ Robert Hamilton, ${ }^{2}$ Shi-Joon Yoo ${ }^{1,2}$

${ }^{1}$ Section of Cardiac Imaging, Department of Diagnostic Imaging, ${ }^{2}$ Division of Cardiology,
Department of Paediatrics, The University of Toronto, Toronto, Canada, ${ }^{3}$ Department of Paediatric Cardiology, RWTH University of Aachen, Aachen, Germany

Lars Grosse-Wortmann was funded by the "Rotationsprogramm" of the Medical Faculty, RWTH Aachen.

Lars Grosse-Wortmann apologises for this error. 